\title{
Meningeal and Bone Metastases from Myoepithelial Carcinoma Ex-pleomorphic Adenoma of the Parotid: CASE REPORT
}

\author{
Razine S, Najem S, Harrak S, Lemsanes S, Benchekroun K, Sninate S, Lkhoyaali S, Mrabti H, \\ Errihani H
}

\begin{abstract}
Myoepithelial carcinomas are rare tumors that make up 1 to 2 percent of all salivary gland neoplasms, that can arise de novo or within a preexisting pleomorphic adenoma.

We report a case of a 58-year-old woman presented with a history of a hard right parotid mass that had gradually increased in size, with subsequent facial paralysis.

A biopsy of the mass was performed, which shows a carcinoma ex-pleomorphic adenoma confirmed by resection of the mass, which showed in immunohistochemistry the presence of a myoepithelial carcinoma of the parotid. The extension assessment revealed the presence of bone and meningeal metastases, contrasting with deterioration in general condition. Chemotherapy was attempted with failure and death of the patient after 2 cycles of carboplatin chemotherapy

Index Terms- myoepithelial carcinoma, parotid, ex-pleomorphic adenoma, bone and meningeal metastases.
\end{abstract}

\section{INTRODUCTION}

Pleomorphic adenoma is well known to be a benign salivary gland tumor, which uncommonly undergoes malignant transformation. When a malignancy arises in pleomorphic adenoma, it is usually carcinoma. Myoepithelial carcinoma of the salivary gland is extremely rare and accounts for less than 1 percent age of all salivary gland tumors. It may develop de novo or may appear in a pre-existing pleomorphic adenoma. [1]

It most commonly affects the parotid gland, but other major or minor salivary glands can also be affected.

The histological features that signify malignancy include tumor infiltration, nuclear atypia, frequent mitosis, and coagulative necrosis [2]

Myepithelial carcinoma typically presents as an asymptomatic mass, until it displays wide growth with subsequent facial paralysis.

\footnotetext{
Razine Sawssane, department of oncology, national institute of oncology, Rabat,Morocco.

Najem Salma, department of oncology, national institute of oncology,Rabat,Morocco.

Harrak Soukaina, department of oncology, national institute of oncology,Rabat,Morocco

Lemsanes S, Benchekroun K, Lkhoyaali S, Mrabti H, Errihani H, department of oncology, national institute of oncology,Rabat,Morocco

Sninate S , department of radiology, national institute of oncology, Rabat, Morocco
}

Patients with MC tend to develop metastasis due to infiltrative, progressive, and locally destructive behavior. Therefore, an early accurate diagnosis and radical surgical treatment for MC should allow for an improved quality of life and increased survival rates. [Three, 4]

Wide surgical excision is the treatment of choice, and radiotherapy is currently recommended for postoperative treatment when a high risk of recurrence or metastasis exists. No standard chemotherapeutic drugs or regimens have yet been established for the treatment of individual types of salivary gland carcinomas. [5]

\section{CASE REPORT}

A 58-year-old woman, without a specific medical or surgical history.

She presented with a swelling of the right parotid region gradually growing over a period of 6 months,associated with diplopia and dizziness

A biopsy of the mass was performed, leading to a diagnosis of myoepithelial carcinoma ex-pleomorphic adenoma:CK7+,CK19+,P63+,PS 100+,AML-EMA-ACE+.

Imaging investigations were then performed. Contrast enhanced computed tomography (CT) showed right parotid process in contact with the carotid artery and presence of secondary locations in the cervical vertebrae, classified T4N0M1.(figure 1-2)

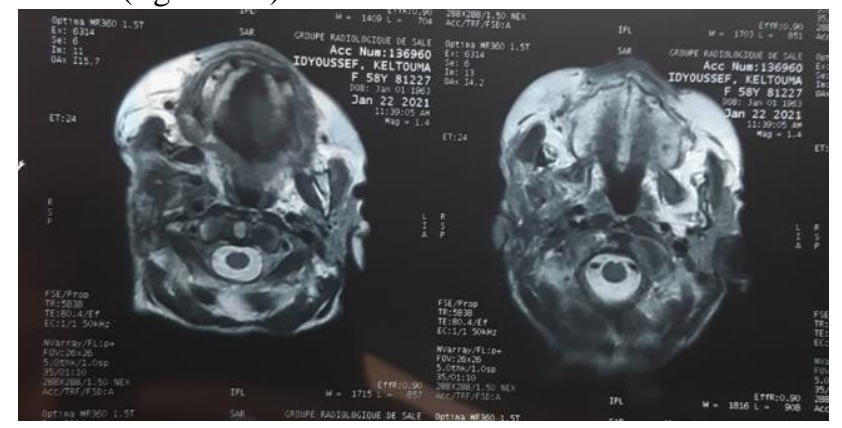

Figure 1: CT image showing right parotid process 


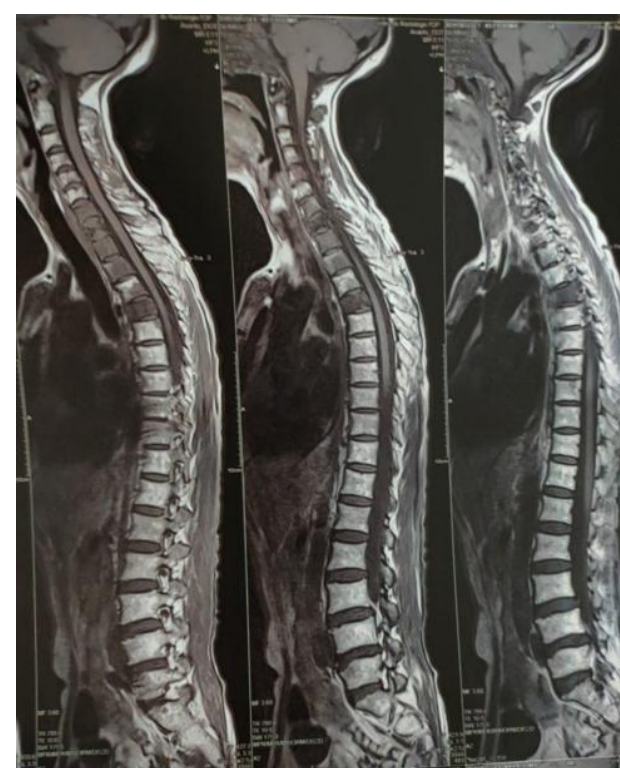

Figure 2:CT image showing vertebral metastases

Parotid tumor exeresis was performed, Postoperative pathology assessment showed a $16 \mathrm{~cm}$ high-grade myoepithelial carcinoma with skin ulceration.

the patient presented facial paralysis after surgery and magnetic resonance imaging (MRI) of the brain was performed and showed meningeal metastases with invasion of the cavernous sinus and the optic foramen responsible for exophthalmos.(figure 3)

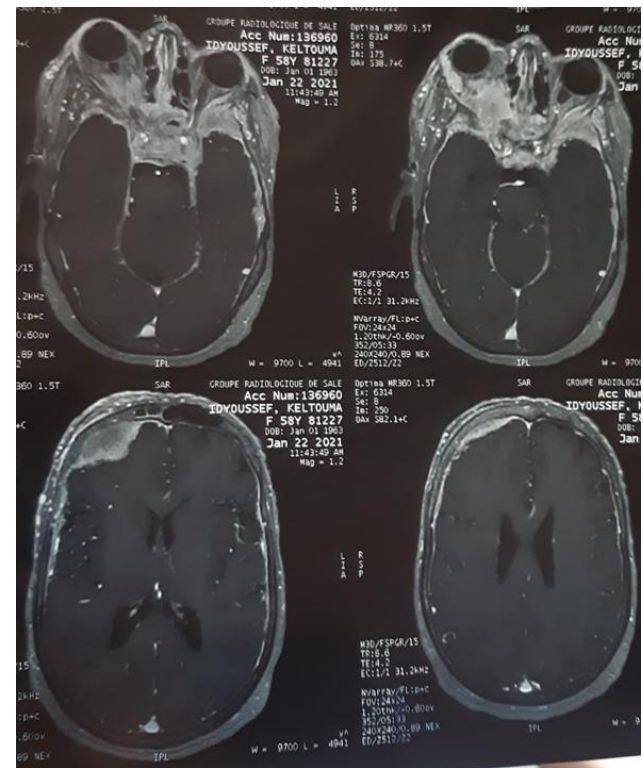

Figure 3: MRI image showing meningeal metastases +exophthalmos

the evolution was marked by alteration in the general condition of the patient, it went from ecog 1 to ecog 3 .

she was refused by radiotherapy, Chemotherapy was attempted with failure and death of the patient after 2 cycles of carboplatin in monotherapy .

\section{DISCUSSION}

Myoepithelial carcinoma of the head and neck is extremely rare. The tumor most frequently develops from the salivary glands in particular, the parotid gland.[6,7].
The majority of the myoepitheliomas described in published reports have been benign, and the malignant counterpart is considered very rare. Malignant myoepitheliomas may appear de novo or develop from a pre-existing pleomorphic adenoma. Grossly, malignant myoepitheliomas range in size from 2 to $20 \mathrm{~cm}$ in the largest dimension.[8]

We herein present our findings of a rare case of a highgrade metastatic myoepithelial carcinoma arising from a pleomorphic adenoma with malignant and metastatic potential. The rarity of this case is that pleomorphic adenoma as a pre-malignancy was observed.

Histologically, the tumor shows morphological heterogeneity and stains positive for vimentin, S 100 protein,cytokeratin, and alpha smooth muscleactin.1-3 Molecular studies have revealed an EWSRI gene translocation in 45 percent of myoepithelial tumors.Interestingly, this gene translocation has also been reportedin rhabdomyosarcomas.[9]

Surgical resection of the tumor is the treatment of choice, while postoperative radiation therapy is recommended for high-risk lesions.Local recurrences following surgery are seen in 30 to 40 percent of patients, with a risk of 30 to 35 percent for metastasis, most commonly to the lungs and then the brain [10]

Nevertheless, chemotherapy and radiotherapy are warranted in patients with highly advanced disease, positive surgical margin, or surgically unresectable disease, although there have been almost no studies on these therapies. Currently, there is no standard therapy for metastatic myoepithelial carcinoma. Surgical resection of metastases, radiotherapy ,and chemotherapy with dacarbazine have been reported in the literature.[6]

DCF(also called TPF) chemotherapy is a regimen combining the $\mathrm{CF}$ regimen and a taxane and has been used for induction chemotherapy for locally advanced or unresectable squamous cell carcinomas of the head and neck. Because improved overall survival has been documented, the TPF regimen has recently been commonly used.[11,12]; In our case, the patient received 2 cycles of carboplatin monotherapy due to her general condition with failure.

Concerning prognosis, it has been described that generally, MC takes a chronic course and approximately $10 \%$ of patients die after recurrence and/or metastases.[13]

\section{CONCLUSION}

$\mathrm{MC}$ of head and neck is a very rare tumor with a high rate of recurrence and metastasis.

Newer strategies or treatments are needed for the future bene $\neg \mathrm{t}$ of patients with advanced disease.

\section{REFERENCES}

[1]. Shinichi Ohba et al.: A case report of metastasizing myoepithelial carcinoma of the parotid gland arising in a recurrent pleomorphic adenoma. 36 (2009) 123-126

[2]. Savera AT, Sloman A, Huvos AG. Myoepithelial carcinoma of the salivary glands: A clinicopathologicstudy of 25 cases. Am J Surg Path 2000;24:761-774 
[3]. Nagao T, Sugano I, Ishida $\mathrm{Y}$, et al. Salivary gland malignant myoepithelioma: a clinicopathologic and immunohistochemical study of ten cases. Cancer 1998; 83: 1292-1299.

[4]. Kane SV and Bagwan IN. Myoepithelial carcinoma of the salivary glands: a clinicopathologic study of 51 cases in a tertiary cancer center. Arch Otolaryngol Head Neck Surg 2010; 136: 702-712.

[5].A.Skalova,K.T.Jakel,Myoepithelialcarcinoma,in:L.Barnes,J.W.Eveson,P .A.Reichartetal.(Eds.),PathologyandGenetics of Head and Neck Tumours.
World
Health
Organization
Classification

ofTumours,Lyon:IARCPress, 2005,pp.240-241.

[6]. Xu T, Liao Z, Tang J, Guo L, et al. Myoepithelial carcinoma of the head and neck: a report of 23 cases and literature review. Cancer TreatCommun. 2014,2(2-3):24-29.

[7].Dimitrijevic MV, Tomanovic NR, Jesic SD, et al.Epithelial-myoepithelial carcinoma-review of linicopathological and immunohistochemical features. Arch Iran Med. 2015;18(4):218-222.

[8].He D, Hua C, Tang X, Feng Y. Cutaneous metastasis from the parotid myoepithelial carcinoma: a case report and review of the literature. J Cutan Pathol. 2008;35(12):1138-1143

[9].Antonescu CR, Zhang L, Chang NE, et al. EWSR1-POU5F1 fusion in soft tissue myoepthilial tumors. A molecular analysis of sixty-six cases, including soft tissues, bone, and visceral lesions, showing common involvement of the EWSR1 gene. Genes Chromosomes.

[10]. Pierard S, Gregoire V, Weynand B, et al: Epithelial-myoepithelial carcinoma of the submandibular gland with symptomatic lung metastases treated with chemotherapy. Eur Arch Otorhinolaryngol 263:1158, 2006

[11].Vermorken JB, Remenar E, van Herpen C, et al: Cisplatin, fluorouracil, and docetaxel in unresectable head and neck cancer. N Engl J Med 357:1695, 2007

[12]. Posner MR, Hershock DM, Blajman CR, et al: Cisplatin and fluorouracil alone or with docetaxel in head and neck cancer.N Engl J Med 357:1705, 2007

[13]. Toida M, Shimokawa K: Epithelial-myoepithelial carcinoma of the parotid gland: Report of a case. J Oral Maxillofac Surg 53:476, 1995 